\title{
Quantification of plasma carnitine and acylcarnitines by high-performance liquid chromatography-tandem mass spectrometry using online solid-phase extraction
}

\author{
Réjane Morand • Massimiliano Donzelli • \\ Manuel Haschke • Stephan Krähenbühl
}

Received: 12 July 2013 /Revised: 12 August 2013 / Accepted: 13 August 2013 / Published online: 1 September 2013

(C) Springer-Verlag Berlin Heidelberg 2013

\begin{abstract}
Carnitine is an amino acid derivative that plays a key role in energy metabolism. Endogenous carnitine is found in its free form or esterified with acyl groups of several chain lengths. Quantification of carnitine and acylcarnitines is of particular interest for screening for research and metabolic disorders. We developed a method with online solid-phase extraction coupled to high-performance liquid chromatography and tandem mass spectrometry to quantify carnitine and three acylcarnitines with different polarity (acetylcarnitine, octanoylcarnitine, and palmitoylcarnitine). Plasma samples were deproteinized with methanol, loaded on a cation exchange trapping column and separated on a reversed-phase C8 column using heptafluorobutyric acid as an ion-pairing reagent. Considering the endogenous nature of the analytes, we quantified with the standard addition method and with external deuterated standards. Solid-phase extraction and separation were achieved within $8 \mathrm{~min}$. Recoveries of carnitine and acylcarnitines were between 98 and $105 \%$. Both quantification methods were equally accurate (all values within 84 to $116 \%$ of target concentrations) and precise (day-to-day
\end{abstract}

Réjane Morand and Massimiliano Donzelli contributed equally to this work.

Electronic supplementary material The online version of this article (doi:10.1007/s00216-013-7309-z) contains supplementary material, which is available to authorized users.

R. Morand $\cdot$ M. Donzelli $\cdot$ M. Haschke $\cdot$ S. Krähenbühl $(\bowtie)$

Clinical Pharmacology and Toxicology, University Hospital Basel,

Spitalstrasse 21/ Petersgraben 4, 4031 Basel, Switzerland

e-mail:kraehenbueh1@uhbs.ch

R. Morand $\cdot$ M. Donzelli $\cdot$ M. Haschke $\cdot$ S. Krähenbühl

Department of Biomedicine, University of Basel, Hebelstrasse 20,

4031 Basel, Switzerland

S. Krähenbühl

Swiss Center for Applied Human Toxicology (SCAHT), University

of Basel, Klingelbergstrasse 61, 4056 Basel, Switzerland variation of less than $18 \%$ ) for all carnitine species and concentrations analyzed. The method was used successfully for determination of carnitine and acylcarnitines in different human samples. In conclusion, we present a method for simultaneous quantification of carnitine and acylcarnitines with a rapid sample work-up. This approach requires small sample volumes and a short analysis time, and it can be applied for the determination of other acylcarnitines than the acylcarnitines tested. The method is useful for applications in research and clinical routine.

Keywords Liquid chromatography/mass spectrometry . Online solid-phase extraction · Carnitine $\cdot$ Acylcarnitines

\section{Introduction}

Carnitine is an endogenous compound that plays a crucial role in energy metabolism. Carnitine is essential for the transport of long-chain fatty acids into the mitochondrial matrix for subsequent $\beta$-oxidation [1] and for the regulation of the cellular coenzyme A (CoA) and acyl-CoA pools $[2,3]$. These functions of carnitine involve the acylation of its $\beta$-hydroxyl group, leading to the formation of various acylcarnitines with different chain lengths [4].

Analytical profiling of carnitine and its acyl derivatives is used for instance to screen newborns and children for metabolic disorders, to monitor patients at risk for or with established hypocarnitinemia [5], and in research projects about energy metabolism [3, 6, 7]. Primary carnitine deficiency is a rare inherited disease which is caused by mutations in the gene coding for OCTN2, the renal carnitine transporter $[8,9]$. Secondary carnitine deficiency is observed more frequently for instance in patients with organic acidurias $[10,11]$ and in patients on hemodialysis [7, 12]. 
Chromatographic methods for the quantification of carnitine and acylcarnitines in plasma use either HPLC or capillary electrophoresis for separation and mostly MS/MS for detection and quantification [13]. Sample preparation can consist of several steps such as solid-phase extraction [14-17] and esterification of carnitine and acylcarnitines at their carboxyl groups with different alcohols to enhance sensitivity $[13,15,18]$. Sample processing is time consuming and imprecisions can potentially arise from incomplete derivatization and/or hydrolysis of acylcarnitines during the derivatization process [19].

Considering the endogenous nature of carnitine and acylcarnitines, their quantification is mainly achieved with surrogate matrices, such as dialyzed plasma or an albumin solution [17] or with surrogate analytes, such as deuterated compounds $[13,15,17]$. Both approaches use substitute parameters for the original matrix or analytes so that comparative studies are required to ensure the validity of the method [20]. In addition, deuterated compounds are expensive and may not be available for all acylcarnitines.

For the analysis of clinical and research samples, time, accuracy, and precision are important factors. Our principal goal was to improve our existing method for plasma carnitine and acylcarnitine analysis [17] by minimizing the time used for sample preparation and by optimizing the quantification procedure. A first specific aim was to establish a simple sample work-up including a precipitation step followed by an online extraction procedure and LC-MS/MS for separation and quantification. A second specific aim was to eliminate the impact of matrix effects on quantification, which can be a problem for carnitine and acylcarnitines in biological matrices $[17,21]$. For that, we compared two methods, namely quantification using deuterated external standards and quantification by the standard addition method (SAM). The newly developed methods were fully validated and compared mutually as well as with the conventional radioenzymatic method [22, 23]. Furthermore, the practicability of the methods was demonstrated by the analysis of clinical samples.

\section{Materials and methods}

Reagents

L-carnitine, L-acetylcarnitine, and L-palmitoylcarnitine were purchased from Sigma (St. Louis, MO, USA). L-octanoylcarnitine was obtained from Dr. H. J. ten Brink (VU Medical Center, Amsterdam, The Netherlands). L-carnitine-d3, L-acetylcarnitine$\mathrm{d} 3$, L-octanoylcarnitine-d3, and L-palmitoylcarnitine-d3 were supplied by Cambridge Isotope Laboratories (Andover, MA, USA).

All other chemicals and solvents were from Merck (Darmstadt, Germany) or Sigma and were of analytical grade.

\section{LC/MS-MS equipment}

The HPLC system consisted of a HTS PAL autosampler (CTC Analytics AG, Zwingen, Switzerland), a column-oven (CTO20A), a quaternary pump (LC-20AD) and a system controller (CBM-20A) from Shimadzu (Reinach, Switzerland). The HPLC system was coupled to an API4000 triple quadrupole mass spectrometer from AB/MDS Sciex (Concord, Canada) with an electrospray ionization source.

Samples were loaded on an Oasis MCX trapping column ( $30 \mu \mathrm{m}, 2.1 \times 20 \mathrm{~mm}$; Waters Corporation, Milford, MA, USA) and separated on a Luna C8 $5 \mu \mathrm{m}$ column $(150 \times 2 \mathrm{~mm})$ equipped with a $\mathrm{C} 8(4 \times 2.0 \mathrm{~mm})$ precolumn (Phenomenex, Torrance, CA, USA).

Sample preparation and carnitine determination by LC/MS-MS

Blood samples were collected in tubes containing lithium heparin. Plasma was obtained after centrifugation at $1,811 \times g$ for $10 \mathrm{~min}$ and stored at $-20^{\circ} \mathrm{C}$ until analysis.

\section{HPLC conditions}

The chromatographic separation was done with a binary flow at $50{ }^{\circ} \mathrm{C}$. Phase A was an aqueous solution containing $5 \mathrm{mmol} / \mathrm{L}$ heptafluorobutyric acid and $5 \mathrm{mmol} / \mathrm{L}$ ammonium acetate and phase B was methanol with the same additives. During $1.5 \mathrm{~min}$, the analytes were loaded on the trapping column using $0.1 \%$ formic acid in water $(V / V)$ as mobile phase (flow, $0.5-1.0 \mathrm{~mL} / \mathrm{min}$ ), whereas the analytical column was conditioned with $10 \%$ phase B (flow, $0.35 \mathrm{~mL} / \mathrm{min}$ ). After valve switching, the analytes were transferred to the analytical column starting at $10 \%$ phase B with a linear increase of the gradient to $95 \%$ phase B within 4 min. After a plateau of $2 \mathrm{~min}$ at $95 \%$ phase B, the analytical column was re-equilibrated for 2 min with $10 \% \mathrm{~B}$. This resulted in a total run time of $8 \mathrm{~min}$. The chromatographic conditions are shown in Electronic supplementary material Fig. S1.

\section{MS conditions}

Carnitine and the acylcarnitines of interest were analyzed in the positive multiple reaction monitoring mode. A first transition was used for quantification, a second one for qualification. Following transitions $(\mathrm{m} / \mathrm{z})$ were used: carnitine, $162 \rightarrow 103$ and $162 \rightarrow 60$; carnitine-d3, $165 \rightarrow 103$ and $165 \rightarrow 63$; acetylcarnitine, $204 \rightarrow 85$ and $204 \rightarrow 145$; acetylcarnitine-d3, 207 $\rightarrow 85$ and $207 \rightarrow 145$; octanoylcarnitine, $288 \rightarrow 85$ and $288 \rightarrow 229$; octanoylcarnitined3, $291 \rightarrow 85$ and $291 \rightarrow 229$; palmitoylcarnitine, $400 \rightarrow 85$ and $400 \rightarrow 341$; and palmitoylcarnitine-d3, $403 \rightarrow 85$ and $403 \rightarrow 341$, respectively. 
The ion spray voltage was $5,500 \mathrm{eV}$; the probe temperature was $450{ }^{\circ} \mathrm{C}$, and the dwell time was $50 \mathrm{~ms}$ for each analyte.

\section{Quantification with the SAM}

Plasma samples to be analyzed were divided in five aliquots of $10 \mu \mathrm{L}$. To each of the $10-\mu \mathrm{L}$ aliquots, 0 to $40 \mu \mathrm{L}$ of standard solution in methanol was added in $10 \mu \mathrm{L}$ steps. The volume of all samples was brought to $50 \mu \mathrm{L}$ with methanol. Standards were prepared in methanol and contained (final concentrations): carnitine, 10-40 $\mu \mathrm{mol} / \mathrm{L}$; acetylcarnitine, 5-20 $\mu \mathrm{mol} / \mathrm{L}$; octanoylcarnitine, $0.25-1.0 \mu \mathrm{mol} / \mathrm{L}$; and palmitoylcarnitine, 0.25-1.0 $\mu \mathrm{mol} / \mathrm{L}$. Afterwards, the plasma samples were deproteinized with the addition of $200 \mu \mathrm{L}$ methanol. The samples were extracted for $10 \mathrm{~min}$ at full speed on a Multi-tube Vortexer (VX-2500, VWR International) and centrifuged at $3,220 \times \mathrm{g}$ for $30 \mathrm{~min}$.

For the determination of total carnitine, acylcarnitines were hydrolyzed under alkaline conditions; $25 \mu \mathrm{L}$ plasma was mixed with $25 \mu \mathrm{L} \mathrm{KOH} 0.5 \mathrm{M}(\mathrm{pH} 13)$ and incubated at $40{ }^{\circ} \mathrm{C}$ for $30 \mathrm{~min}$. The mixture was then neutralized with $50 \mu \mathrm{L}$ at $1 \%$ formic acid in water $(V / V)$ and centrifuged for $10 \mathrm{~min}$ at $1,811 \times \mathrm{g}$.

After quantification, the LC/MS-MS peak areas were fitted to the added concentrations and the endogenous concentration was calculated as the intercept of this curve on the $x$-axis.

Quantification with external deuterated standards (deuterated standard method)

For this type of quantification, we constructed an external calibration curve using the peak areas from deuterated standards in pooled plasma. Pooled plasma was spiked with deuterated compounds in the following concentration ranges (final concentrations): carnitine-d3, 1-100 $\mu \mathrm{mol} / \mathrm{L}$; acetylcarnitine$\mathrm{d} 3,0.25-50 \mu \mathrm{mol} / \mathrm{L}$; octanoylcarnitine-d3, 0.025-10 $\mu \mathrm{mol} / \mathrm{L}$; and palmitoylcarnitine, $0.05-10 \mu \mathrm{mol} / \mathrm{L}$. These plasma samples $(10 \mu \mathrm{L})$ were then mixed with $200 \mu \mathrm{L}$ methanol. After $10 \mathrm{~min}$ shaking, the samples were centrifuged at 3,220×g for $30 \mathrm{~min}$ and analyzed as described above.

Unspiked plasma samples were prepared identically and the analyte concentration in these samples was calculated using the calibration curve obtained with the deuterated standards. The sample and standard work-up for total carnitine was identical as described for the SAM.

\section{Quantification with the radioenzymatic method}

The radioenzymatic method was used for hydrolyzed samples as a comparison with the two LC/MS-MS methods. The radioenzymatic method used by us [23] is based on the method described by Brass and Hoppel [22].

\section{Validation of the method}

We determined accuracy and precision of both the SAM and the deuterated standard method (DSM). Given the endogenous nature of carnitine and its derivatives and the lack of blank plasma, we used spiked plasma samples for these determinations.

For the validation of the SAM, pooled plasma was spiked with three concentrations of carnitine, acetylcarnitine, octanoylcarnitine, and palmitoylcarnitine (low-, medium-, and high-quality controls (QC)). These samples contained the unknown endogenous concentration of the pooled plasma plus $12.5,25$, or $37.5 \mu \mathrm{mol} / \mathrm{L}$ carnitine; 3,6 , or $9 \mu \mathrm{mol} / \mathrm{L}$ acetylcarnitine; $0.03,0.06$, or $0.09 \mu \mathrm{mol} / \mathrm{L}$ octanoylcarnitine; and $0.125,0.25$, or $0.375 \mu \mathrm{mol} / \mathrm{L}$ palmitoylcarnitine, respectively.

For the validation of the DSM, pooled plasma was spiked with three concentrations of deuterated carnitine, acetylcarnitine, octanoylcarnitine, and palmitoylcarnitine. The added concentrations were $1.25,12.5$, or $125 \mu \mathrm{mol} / \mathrm{L}$ for carnitine-d3; $0.4,4$, or $40 \mu \mathrm{mol} / \mathrm{L}$ for acetylcarnitine-d3; $0.08,0.8$, or $8 \mu \mathrm{mol} / \mathrm{L}$ for octanoylcarnitine-d 3 ; and $0.08,0.8$, or $8 \mu \mathrm{mol} / \mathrm{L}$ for palmitoylcarnitine.

For total carnitine, the samples were hydrolyzed and the concentration determined using the SAM. For comparison, the same samples were also determined using the radioenzymatic assay [22, 23].

Recovery was assessed with three concentrations of the four analytes spiked before or after the protein precipitation step. For the assessment of short-term stability, samples were spiked, stored at room temperature for $8 \mathrm{~h}$, analyzed, and compared with freshly spiked samples. For stability under storage conditions, samples were spiked and kept frozen at $-20{ }^{\circ} \mathrm{C}$ for 1 week. The samples were then analyzed and compared with freshly spiked plasma samples. For freeze/thaw stability, freshly prepared samples were frozen at $-20{ }^{\circ} \mathrm{C}$ for at least $12 \mathrm{~h}$ and then thawed at room temperature for $30 \mathrm{~min}$. After three freeze-thaw cycles, the samples were analyzed and compared with freshly prepared samples. To determine the stability of the analytes in solution, freshly made stock solutions were analyzed and compared with stock solutions that had been stored at $-20^{\circ} \mathrm{C}$ for 4 months.

The lower limit of quantification was defined as the lowest concentration measured with precision and accuracy not exceeding $20 \%$ with a signal to noise ratio of $>10$.

Source of biological samples

To test the practical application of the method, two types of plasma samples were obtained and analyzed. Samples were obtained from two infants with medium-chain acyl-CoA dehydrogenase deficiency and from a healthy 58-year-old man exercising until exhaustion. Exhaustive exercise was 
performed on a cycling ergometer, starting at a workload of $220 \mathrm{~W}$ with increases of $20 \mathrm{~W}$ every $2 \mathrm{~min}$. Exhaustion was achieved after having finished the $380 \mathrm{~W}$ workload phase. Samples were obtained before exercise, in the middle of every workload phase, and during the recovery phase up to $15 \mathrm{~min}$ after termination of exercise.

\section{Results and discussion}

Probe preparation and analyte separation

A first aim of the current project was to develop a rapid and reliable method with a one-step sample preparation that could potentially be automated. Such methods usually have an initial protein precipitation step which is followed by direct application of the supernatant on the HPLC system and on-column clean-up of the sample. Online solid-phase extraction coupled to chromatographic separation in one run conciliates the advantages of solid-phase extraction with time efficiency. In several publications, online solid-phase extraction followed by LC-MS has been described for the analysis of carnitine and certain acylcarnitines. Ho et al. [21] reported a procedure for the analysis of free and total carnitine, whereas Hirche et al. [24] described a method for the analysis of carnitine, acetylcarnitine, and propionylcarnitine and for the carnitine precursors butyrobetaine and trimethyllysine. Goshal et al. [25] published a method for the analysis of carnitine and acylcarnitines from $\mathrm{C} 2$ to $\mathrm{C} 18$ and at the same time demonstrated their clinical applicability. The run time of the HPLC step was $17 \mathrm{~min}$ for this method, however, which we considered too long. As shown previously [16], the addition of heptafluorobutyric acid as an ion-pairing agent to the HPLC solvents results in sharper peaks and allows achieving a good separation. We followed this strategy and achieved a sufficient separation of carnitine, butyrobetaine, and acylcarnitines from $\mathrm{C} 2$ to $\mathrm{C} 18$ within $6 \mathrm{~min}$, resulting in a total run time of $8 \mathrm{~min}$ (Fig. 1; Electronic supplementary material (ESM) Fig. S2). This run time is longer than the $4.5 \mathrm{~min}$ described by Ho et al. [21], but Ho et al. only analyzed free and total carnitine and not acylcarnitines. In comparison, run times were between 16 and $75 \mathrm{~min}$ in other published methods analyzing carnitine and acylcarnitines [14, 15, 17, 25].

Offline solid-phase extraction after the initial protein precipitation step is a frequently used alternative $[14-17,26]$ for the online procedure that we used in the current study. As carnitine and acylcarnitines are cations under acidic conditions, matrices with cation exchange properties are preferred to optimize retention $[15,17]$. This is the reason why we also used a cation exchanger for online sample clean-up in the current study. While offline solid-phase extraction can enhance the sensitivity of the chromatographic assays, the process is usually time-consuming and difficult to automate.
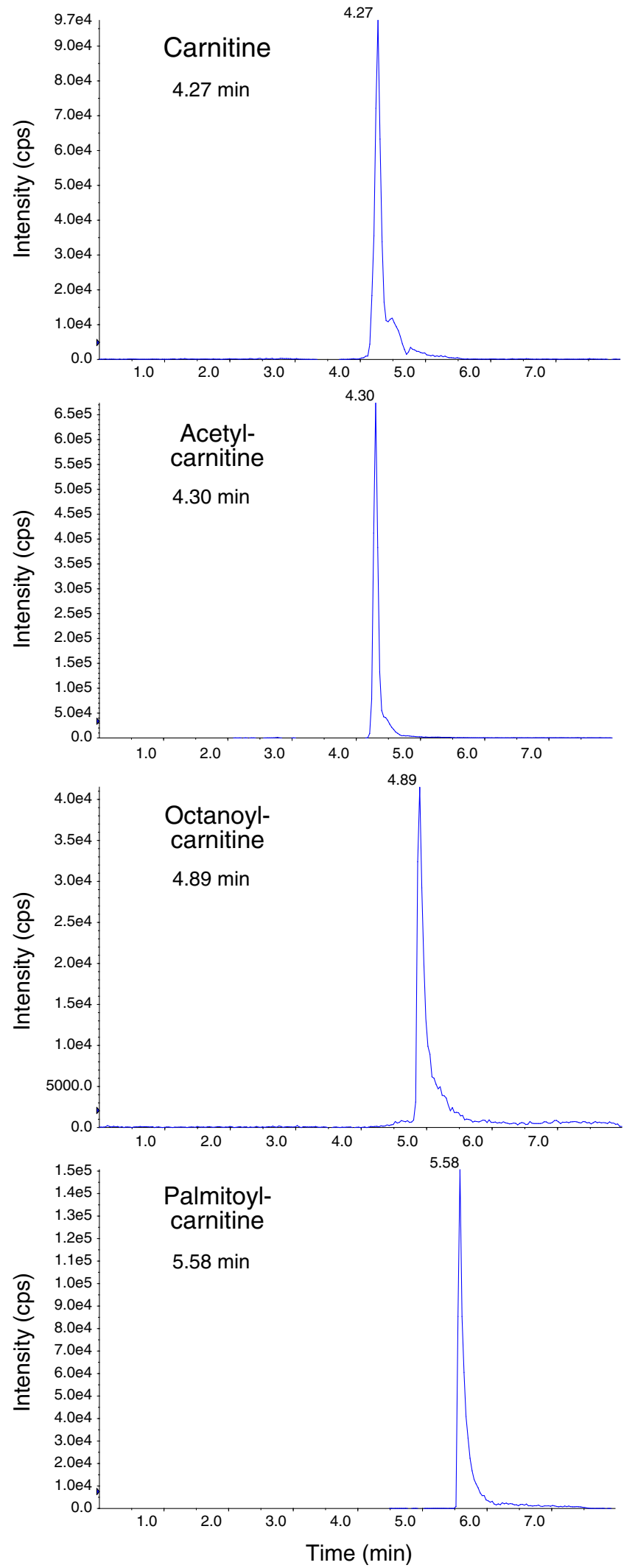

Fig. 1 Representative LC-MS/MS multiple reaction monitoring chromatograms of carnitine, acetylcarnitine, octanoylcarnitine, and palmitoylcarnitine standards. HPLC and MS conditions are described in the "Materials and methods" 
As already published previously $[16,17]$, we prefer to analyze carnitine and acylcarnitines without prior derivatization. Frequently used derivatization procedures include the formation of the methyl [13] or the butyl ester [18] as well as the formation of the pentafluorophenacyl ester [15]. These procedures use more time for sample preparation than a simple precipitation step, can therefore be less well automated and butylation has the disadvantage that acylcarnitines may be hydrolyzed during esterification [19]. Conversely, they have the advantage to be more specific for the separation of acylcarnitines with the same molecular mass [15].

Recovery and stability of the analytes

After having demonstrated a good separation for carnitine and the most important acylcarnitines between $\mathrm{C} 2$ and $\mathrm{C} 18$, we concentrated on the analysis of carnitine, acetylcarnitine, octanoylcarnitine and palmitoylcarnitine. As shown in Table 1, the recoveries of these four analytes were between 98 and $106 \%$. This is similar to the methods of Goshal et al. [25] and Minkler et al. [15], who reported recoveries in the range of 84 and 112 and 83 to $107 \%$, respectively, for these compounds. After addition of these four analytes to pooled plasma, they were still stable after three freeze-thaw cycles. After 1 week at $-20{ }^{\circ} \mathrm{C}$, acetylcarnitine and octanoylcarnitine showed a drop by 7.4 and $11.4 \%$, respectively. Similarly, after $8 \mathrm{~h}$ at room temperature, acetylcarnitine and octanoylcarnitine had decreased by 5.9 and $6.4 \%$, respectively, whereas carnitine and palmitoylcarnitine remained stable (Table 1).

\section{Quantification and method validation}

A second aim was to develop a method minimizing the impact of matrix effects on quantification of carnitine and acylcarnitines. We first tested the feasibility of a classical quantification with standard curves prepared in carnitine-free matrices such as water or $4 \%$ bovine serum albumin (BSA). As shown in ESM Fig. S3A-D, the slopes of the standard curves in water containing BSA were different from the slopes in pooled plasma for carnitine and acetylcarnitine, indicating a significant matrix effect. Matrix effects have also been described in the publication of Ho et al. [21] who analyzed free and total carnitine after protein precipitation with acetonitrile, and in the publication of Vernez et al. [17] who analyzed carnitine and acylcarnitines after protein precipitation and solid-phase extraction. An alternative could be the use of denatured matrices such as dialyzed plasma [17, 27]. Dialysis of plasma is time consuming, however, and the absence of matrix effects should be demonstrated for every batch. We therefore decided to validate and compare two different methods of quantification, namely the SAM and the use of external deuterated standards (DSM), which both account for the observed matrix effect.

Accuracy of the two methods was assessed using three QCs measured on three different days. The QCs were generated from pooled plasma, which was spiked with three known concentrations (low, medium, and high concentration) of the four analytes investigated (Table 2). The target values were calculated by adding the spiked to the respective endogenous concentration of the individual analytes in the pooled plasma. For the SAM, the measured concentrations were within $\pm 7.5 \%$ of the expected concentrations for total carnitine and carnitine in the entire concentration range tested. For acetylcarnitine, octanoylcarnitine, and palmitoylcarnitine, the variability was higher though not exceeding $15.4 \%$ deviation from the target values. For the DSM, the accuracy was in the same range as for the SAM. The concentrations measured were within $13 \%$ of the expected values for the medium and high concentrations and within $16 \%$ for the low concentrations for all analytes.

The precision of the methods was determined using the three QCs at different concentrations (low, medium, and high) as described for the determination of the accuracy (Table 2) as well as using plasma samples of six healthy volunteers (results not shown). These samples were analyzed with both quantification methods on three different days. For the SAM, the mean interday variation was below $10 \%$ for free carnitine, acetylcarnitine, octanoylcarnitine, palmitoylcarnitine, and total carnitine. None of the individual coefficients of variation exceeded $17.7 \%$. For the DSM, the interday precision was within $7.5 \%$ for all analytes in the entire concentration range tested.

As shown for carnitine and octanoylcarnitine in ESM Fig. S4, both methods were linear within the analyzed range with coefficients of correlation of $>0.99$. Between the results

Table 1 Recovery and stability of the analytes

\begin{tabular}{lcccrr}
\hline Carnitine species & Recovery (\%) & Freeze-thaw stability (\%) & 1 week stability (\%) & 8 h stability (\%) & Stability of stock solution (\%) \\
\hline Carnitine & $100.6 \pm 0.7$ & $103.2 \pm 1.5$ & $95.2 \pm 4.1$ & $103.1 \pm 5.5$ & $99.0 \pm 16.3$ \\
Acetylcarnitine & $98.4 \pm 2.7$ & $105.3 \pm 2.7$ & $92.6 \pm 3.7$ & $94.1 \pm 2.3$ & $102.8 \pm 16.5$ \\
Octanoylcarnitine & $102.3 \pm 3.8$ & $103.7 \pm 3.7$ & $89.6 \pm 7.1$ & $93.6 \pm 2.9$ & $110.0 \pm 24.9$ \\
Palmitoylcarnitine & $105.6 \pm 2.2$ & $103.6 \pm 4.1$ & $95.8 \pm 1.2$ & $98.7 \pm 6.3$ & $99.2 \pm 27.8$ \\
\hline
\end{tabular}

Stability studies of the four analytes were performed on seven replicates of the three quality controls. Values are given as mean \pm coefficient of variation (standard deviation expressed as a percentage of the mean) 
Table 2 Accuracy and precision of the methods used for quantification

\begin{tabular}{|c|c|c|c|c|c|c|}
\hline \multirow[t]{2}{*}{ Carnitine species } & \multicolumn{2}{|l|}{ QCL } & \multicolumn{2}{|l|}{ QCM } & \multicolumn{2}{|l|}{ QCH } \\
\hline & SAM & DSM & SAM & DSM & SAM & DSM \\
\hline \multicolumn{7}{|l|}{ Carnitine } \\
\hline Precision (CV \%) & 3.8 & 5.9 & 11.7 & 0.9 & 10.0 & 4.0 \\
\hline Accuracy $(\%$ expected $)$ & 106 & 108 & 103 & 112 & 101 & 108 \\
\hline \multicolumn{7}{|l|}{ Acetylcarnitine } \\
\hline Precision (CV \%) & 0.3 & 7.5 & 8.2 & 0.9 & 5.3 & 1.9 \\
\hline Accuracy (\% expected) & 95.2 & 99.8 & 90.4 & 110 & 84.6 & 110 \\
\hline \multicolumn{7}{|l|}{ Octanoylcarnitine } \\
\hline Precision (CV \%) & 12.8 & 3.9 & 17.7 & 2.9 & 14.3 & 1.1 \\
\hline Accuracy (\% expected) & 108 & 116 & 90.4 & 111 & 99.1 & 113 \\
\hline \multicolumn{7}{|l|}{ Palmitoylcarnitine } \\
\hline Precision (CV \%) & 9.5 & 0.9 & 10.4 & 5.4 & 10.9 & 5.9 \\
\hline Accuracy $(\%$ expected $)$ & 94.8 & 116 & 85.6 & 98.4 & 86.8 & 107 \\
\hline \multicolumn{7}{|l|}{ Total carnitine } \\
\hline Precision (CV \%) & 8.4 & 3.4 & 16.9 & 4.9 & 7.9 & 6.6 \\
\hline Accuracy (\% expected) & 98 & 103 & 92.5 & 92.8 & 98.4 & 100 \\
\hline
\end{tabular}

Both accuracy and precision were assessed with three quality controls (QC; low (QCL), medium (QCM), and high $(\mathrm{QCH})$ ) generated from pooled plasma spiked with three different concentrations of carnitine and three acylcarnitines. The expected concentration was calculated from the concentration measured in pooled plasma plus the spiked concentrations. The precision is expressed as coefficient of variation $(\mathrm{CV}$; standard deviation expressed as a percentage of the mean). The accuracy is given as the ratio of the measured and the expected concentration expressed as a percentage

$S A M$ standard addition method, DSM deuterated standard method generated with the SAM and the DSM, we found correlations ranging from 0.720 to 0.981 . For carnitine we observed a trend to higher values with the DSM, whereas for the other analytes, the results were comparable (Fig. 2).

Regarding the sensitivity of our method, we defined the lower limits of quantification (LLOQ) as the lowest concentration of the respective deuterated standard measured with reliable precision and accuracy. For octanoylcarnitine, the LLOQ was $25 \mathrm{nmol} / \mathrm{L}$ and for palmitoylcarnitine $50 \mathrm{nmol} / \mathrm{L}$. LLOQ for carnitine and acetylcarnitine was $250 \mathrm{nmol} / \mathrm{L}$. These values are in the same range as reported by Ho et al. [21] and by Hirche et al. [24].

The total carnitine concentration of a sample (the carnitine concentration after hydrolysis of all acylcarnitines) provides valuable information about the size of the carnitine pool and the quality of the analysis of the individual carnitine species (the sum of the individual carnitine species should equal the total carnitine concentration). We therefore perform the determination of the total carnitine concentration routinely in all samples that we analyze. Regarding the importance of this determination, we assessed the total carnitine concentration using both the SAM as well as the DSM and compared the values with the radioenzymatic method. As shown in ESM Table S1, there was a good agreement between the methods used. The average of the largest difference between the values obtained was $6.7 \%$ (range, 3.2 to $9.9 \%$ ) of the mean value. The mean intercept of the correlations was 0.97 (range, -1.4 to 3.1), the mean slope was 0.958 (range, 0.918 to 1.016 ), and the mean $r^{2}$ was 0.982 (range, 0.975 to 0.994 ).
Fig. 2 Carnitine (a) acetyl- (b), octanoyl- (c), and palmitoylcarnitine (d) concentrations were measured with the standard addition method and with the deuterated standard method. Both methods are described in the "Materials and methods." The two methods were compared using linear regression. Concentrations are given in micromoles per liter a

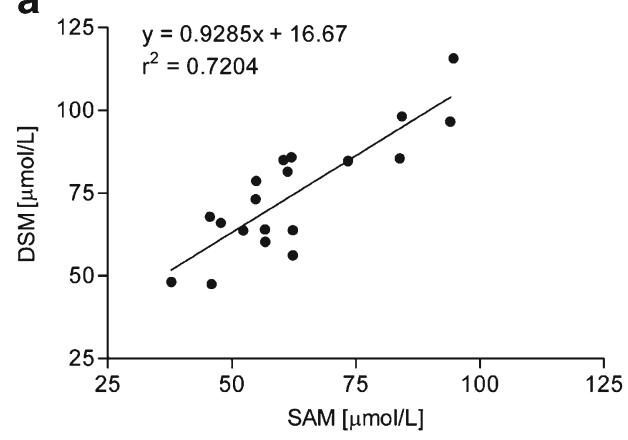

C

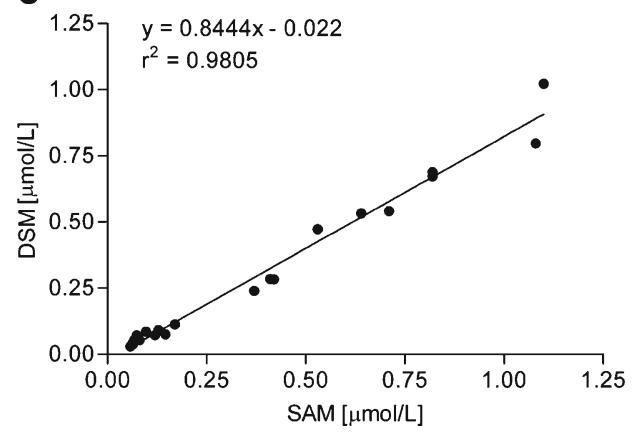

b

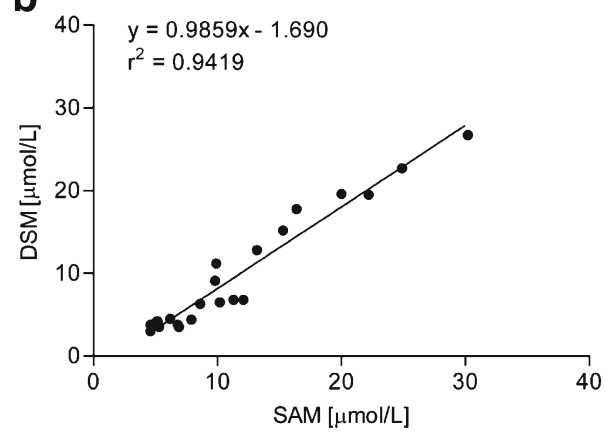

d

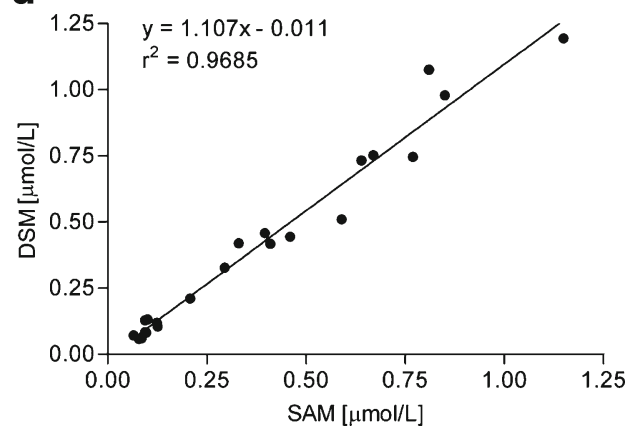


Table 3 Plasma concentrations of carnitine and acylcarnitines in two patients with MCAD deficiency

\begin{tabular}{|c|c|c|c|c|c|c|}
\hline & \multicolumn{2}{|c|}{ Patient 1} & \multicolumn{2}{|c|}{ Patient 2} & \multicolumn{2}{|c|}{ Reference values } \\
\hline & SAM & DSM & SAM & DSM & SAM & DSM \\
\hline Carnitine & 37.8 & 48.2 & 45.9 & 47.5 & $56.4 \pm 5.5$ & $65.4 \pm 7.7$ \\
\hline Acetylcarnitine & 11.3 & 6.8 & 6.8 & 3.8 & $5.9 \pm 1.5$ & $4.2 \pm 0.8$ \\
\hline Octanoylcarnitine & 1.1 & 1.0 & 4.2 & 4.7 & $0.08 \pm 0.03$ & $0.05 \pm 0.02$ \\
\hline Palmitoylcarnitine & 0.59 & 0.51 & 0.10 & 0.13 & $0.10 \pm 0.02$ & $0.08 \pm 0.03$ \\
\hline
\end{tabular}

The carnitine concentrations were measured with both the standard addition method (SAM) and deuterated standard method (DSM). Concentrations are given in micromoles per liter. Reference values from healthy volunteers $(n=10)$ are given as mean $\pm \mathrm{SD}$

Comparison of the standard addition and the DSM

The SAM, which allows the quantification of the analytes in the native matrix, completely eliminates the impact of matrix effects on quantification. A disadvantage of this method is that a single sample has to be analyzed five times to obtain a reliable curve which can be used for quantification. Taking into account the short analysis time, this disadvantage appears to be acceptable. As the same standard solution in different volumes is added to the plasma aliquots, a semi-automated sample processing is possible. Together with the 30-min hydrolysis time for the determination of total carnitine, mixing and centrifugation steps, the analysis of one sample for free and total carnitine and acylcarnitines lasts approximately $1.5 \mathrm{~h}$ and approximately $15 \mathrm{~h}$ for ten samples.

The DSM also minimizes the impact of matrix effects on quantification and had a similar accuracy and precision compared with the SAM. As two standard curves have to be run (one for the hydrolyzed and one for the nonhydrolyzed samples), the analysis of one sample takes approximately $2 \mathrm{~h}$. For each new sample, $20 \mathrm{~min}$ more are needed, resulting in $5 \mathrm{~h}$ for ten samples. If more than one sample has to be analyzed, the DSM is therefore faster than the SAM. Disadvantages of the DSM are higher costs for the deuterated standards and the possible lack of appropriate standards for specific acylcarnitines.

\section{Application of the method to biological samples}

The SAM was applied for the quantification of carnitine and three acylcarnitines in plasma samples of two patients with medium-chain acyl-CoA dehydrogenase (MCAD) deficiency and the DSM for the analysis of plasma samples from a healthy volunteer completing a cycling exercise until exhaustion.

As expected, the two patients with MCAD deficiency had elevated plasmatic octanoylcarnitine concentrations $[18,26]$. In the first patient, the octanoylcarnitine concentration was more than ten times higher than in healthy volunteers and in the second patient approximately 50 times higher. In the first patient, also the palmitoylcarnitine concentration was elevated while this was normal in the second patient. Carnitine and acetylcarnitine concentrations were in a normal range in both patients (Table 3).

In the healthy volunteer cycling until exhaustion, we observed increasing acetylcarnitine concentrations with increasing workload. In comparison, the free carnitine concentrations first slightly increased with exercise and then decreased in parallel with increasing acetylcarnitine concentrations at higher exercise intensities (Fig. 3). Increasing acetylcarnitine concentrations in humans exercising above the lactate threshold have been described earlier [3].

The two examples show that the method can be used reliably for the quantification of carnitine and acylcarnitines in patient samples. Further investigations have shown that the method can easily be adapted for the analysis of other acylcarnitines than those quantified in this study and is also suitable for other matrices such as skeletal muscle and urine (data not shown).
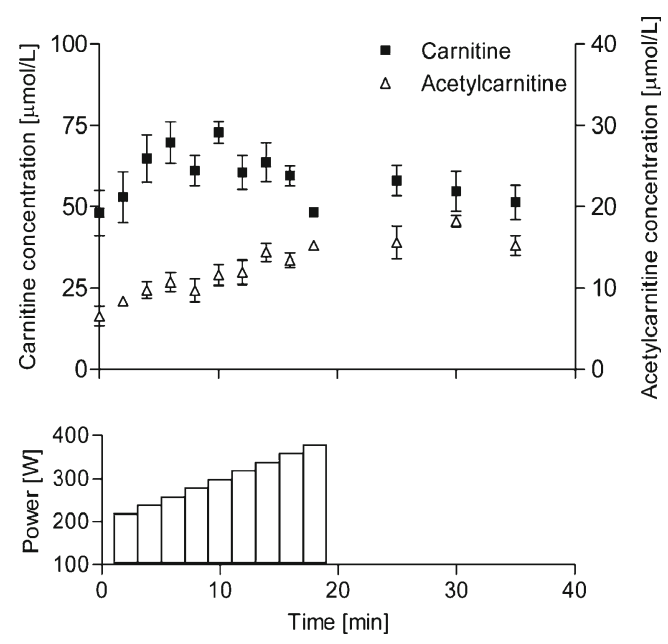

Fig. 3 The deuterated standard method was used for the quantification of carnitine and acetylcarnitine in plasma samples of a healthy male volunteer completing a cycle exercise with increasing workload until exhaustion. The workload started at $220 \mathrm{~W}$ and was increased by $20 \mathrm{~W}$ every $2 \mathrm{~min}$. Exhaustion was reached after $18 \mathrm{~min}$ at $380 \mathrm{~W}$. Mean concentrations in micromoles per liter $\pm \mathrm{SD}$ of three determinations are shown 


\section{Conclusions}

In conclusion, we describe a fast and reliable method for the analysis of carnitine and acylcarnitines requiring small sample volumes and, in the case of the deuterated method, a short total analysis time. Our approach requires minimal sample work-up and can therefore be automated. The method is useful for research projects with large numbers of specific analyses but also for clinical applications.

Acknowledgments The authors would like to thank Dr. Jean-Marc Nuoffer from the Inselspital in CH-3010 Berne for providing the two patient samples.

Financial support This study was supported by a grant from the Swiss National Science Foundation to SK (SNF 31003A-132992).

\section{References}

1. Fritz IB (1955) The effects of muscle extracts on the oxidation of palmitic acid by liver slices and homogenates. Acta Physiol Scand 34:367-385

2. Brass EP, Hoppel CL (1980) Relationship between acid-soluble carnitine and coenzyme A pools in vivo. Biochem J 190:495-504

3. Friolet R, Hoppeler H, Krahenbuhl S (1994) Relationship between the coenzyme A and the carnitine pools in human skeletal muscle at rest and after exhaustive exercise under normoxic and acutely hypoxic conditions. J Clin Invest 94:1490-1495

4. Steiber A, Kerner J, Hoppel CL (2004) Carnitine: a nutritional, biosynthetic, and functional perspective. Mol Aspects Med 25:455-473

5. Millington DS, Kodo N, Norwood DL, Roe CR (1990) Tandem mass spectrometry: a new method for acylcarnitine profiling with potential for neonatal screening for inborn errors of metabolism. J Inherit Metab Dis 13:321-324

6. Morand R, Todesco L, Donzelli M, Fischer-Barnicol D, Mullen PJ, Krahenbuhl S (2012) Effect of short- and long-term treatment with valproate on carnitine homeostasis in humans. Ther Drug Monit 34: 406-414

7. Vernez L, Dickenmann M, Steiger J, Wenk M, Krahenbuhl S (2006) Effect of L-carnitine on the kinetics of carnitine, acylcarnitines and butyrobetaine in long-term haemodialysis. Nephrol Dial Transplant $21: 450-458$

8. Tamai I, Ohashi R, Nezu J, Yabuuchi H, Oku A, Shimane M, Sai Y, Tsuji A (1998) Molecular and functional identification of sodium iondependent, high affinity human carnitine transporter OCTN2. J Biol Chem 273:20378-20382

9. Wang Y, Ye J, Ganapathy V, Longo N (1999) Mutations in the organic cation/carnitine transporter OCTN2 in primary carnitine deficiency. Proc Natl Acad Sci U S A 96:2356-2360

10. Chalmers RA, Roe CR, Stacey TE, Hoppel CL (1984) Urinary excretion of $\mathrm{L}$-carnitine and acylcarnitines by patients with disorders of organic acid metabolism: evidence for secondary insufficiency of L-carnitine. Pediatr Res 18:1325-1328

11. Di Donato S, Rimoldi M, Garavaglia B, Uziel G (1984) Propionylcarnitine excretion in propionic and methylmalonic acidurias: a cause of carnitine deficiency. Clin Chim Acta 139:13-21
12. Hiatt WR, Koziol BJ, Shapiro JI, Brass EP (1992) Carnitine metabolism during exercise in patients on chronic hemodialysis. Kidney Int 41:1613-1619

13. Millington DS, Stevens RD (2011) Acylcarnitines: analysis in plasma and whole blood using tandem mass spectrometry. Methods Mol Biol 708:55-72

14. Maeda Y, Ito T, Suzuki A, Kurono Y, Ueta A, Yokoi K, Sumi S, Togari H, Sugiyama N (2007) Simultaneous quantification of acylcarnitine isomers containing dicarboxylic acylcarnitines in human serum and urine by high-performance liquid chromatography/electrospray ionization tandem mass spectrometry. Rapid Commun Mass Spectrom 21: 799-806

15. Minkler PE, Stoll MS, Ingalls ST, Yang S, Kerner J, Hoppel CL (2008) Quantification of carnitine and acylcarnitines in biological matrices by HPLC electrospray ionization-mass spectrometry. Clin Chem 54:1451-1462

16. Vernez L, Hopfgartner G, Wenk M, Krahenbuhl S (2003) Determination of carnitine and acylcarnitines in urine by high-performance liquid chromatography-electrospray ionization ion trap tandem mass spectrometry. J Chromatogr A 984:203-213

17. Vernez L, Wenk M, Krahenbuhl S (2004) Determination of carnitine and acylcarnitines in plasma by high-performance liquid chromato graphy/electrospray ionization ion trap tandem mass spectrometry. Rapid Commun Mass Spectrom 18:1233-1238

18. Chace DH, Hillman SL, Van Hove JL, Naylor EW (1997) Rapid diagnosis of MCAD deficiency: quantitative analysis of octanoylcarnitine and other acylcarnitines in newborn blood spots by tandem mass spectrometry. Clin Chem 43:2106-2113

19. Johnson DW (1999) Inaccurate measurement of free carnitine by the electrospray tandem mass spectrometry screening method for blood spots. J Inherit Metab Dis 22:201-202

20. Chau CH, Rixe O, McLeod H, Figg WD (2008) Validation of analytic methods for biomarkers used in drug development. Clin Cancer Res 14:5967-5976

21. Ho CS, Cheng BS, Lam CW (2003) Rapid liquid chromatographyelectrospray tandem mass spectrometry method for serum free and total carnitine. Clin Chem 49:1189-1191

22. Brass EP, Hoppel CL (1978) Carnitine metabolism in the fasting rat. J Biol Chem 253:2688-2693

23. Heuberger W, Berardi S, Jacky E, Pey P, Krahenbuhl S (1998) Increased urinary excretion of carnitine in patients treated with cisplatin. Eur J Clin Pharmacol 54:503-508

24. Hirche F, Fischer M, Keller J, Eder K (2009) Determination of carnitine, its short chain acyl esters and metabolic precursors trimethyllysine and gamma-butyrobetaine by quasi-solid phase extraction and MS/MS detection. J Chromatogr B: Analyt Technol Biomed Life Sci 877: $2158-2162$

25. Ghoshal AK, Guo T, Soukhova N, Soldin SJ (2005) Rapid measurement of plasma acylcarnitines by liquid chromatography-tandem mass spectrometry without derivatization. Clin Chim Acta 358: 104-112

26. Yokoi K, Ito T, Maeda Y, Nakajima Y, Ueta A, Nomura T, Koyama N, Kato I, Suzuki S, Kurono Y, Sugiyama N, Togari H (2007) Acylcarnitine profiles during carnitine loading and fasting tests in a Japanese patient with medium-chain acyl-CoA dehydrogenase deficiency. Tohoku J Exp Med 213:351-359

27. Liu Z, Mutlib AE, Wang J, Talaat RE (2008) Liquid chromatography/ mass spectrometry determination of endogenous plasma acetyl and palmitoyl carnitines as potential biomarkers of beta-oxidation in mice. Rapid Commun Mass Spectrom 22:3434-3442 